\title{
Simulation Analysis on Partial Discharge Signals Produced by Multiple Insulation Defects in GIS
}

\author{
Yuxin Yun*, Guangke Xu, Weiwei Zhang, Xing Li and Lingying Chen \\ State Grid Shandong Electric Power Research Institute, Jinan, China
}

\begin{abstract}
In this paper, the simulation analysis on partial discharge (PD) signals produced by multiple insulation defects in gas insulated substation (GIS) has been researched by the simulation software of electromagnetic field (XFDTD). The theoretical analysis and simulation results show that the single partial discharge signal is the linear convolution of the partial discharge current signal and its corresponding impact response in GIS. The mixed partial discharge signals are the linear mixtures of every partial discharge signal.
\end{abstract}

\section{Introduction}

Gas insulated substation (GIS) has been widely used in the power system and the researches on the partial discharge (PD) in GIS have been widely concerned. The PD researches mainly focus on the single PD signal propagation and $\mathrm{PD}$ fault location at present [1-3]. However, the researches on the propagation characteristics and pattern recognition of ultra-high frequency (UHF) PD signals produced by multiple insulation defects in GIS are rare, and they gradually become a hot topic.

The mixed signals acquired by the UHF sensors are the superposition of each single PD signal (source signal) and noise. There are mainly two kinds of mixed forms: first, each source signal propagates separately, and is captured by the same sensor after being contaminated with noise; second, some source signals are propagated. After the noise is mixed with another part of the source signal, it is then propagated and captured by the sensor [3-6]. In the first form, it is very difficult to select features and the rich experience of data processing is needed. The second form gradually becomes a hot topic because of clear thinking and direction. In reference [7], wavelet transform is used to analyze and identify the types of insulation defects corresponding to mixed PD signals produced by multiple insulation defects in transformers. In reference [8], the second-order nonstationary source (sons) algorithm is used to separate the mixed PD signals in GIS, which provides a new direction for pattern recognition of multiple insulation defects. However, the algorithm of sons and wavelet transform is too complex to deal with a large number of data and is not conducive to practical application.

In this paper, firstly, the theoretical analysis and formula derivation on the mixed modes of PD signals are conducted by the antenna theory. Secondly, the reverse process of PD signal mixing process is discussed by the blind source separation method. Finally, the electromagnetic simulation software XFDTD is used to verify the conclusions.

\section{Mixed relationships of PD signals}

\subsection{Linear Mixing}

The nature of GIS PD fault is that the insulating medium will be punctured under abnormal high field strength, and then a discharge current path is formed in GIS. So, a high-frequency pulse current signals is generated. The discharge current path can act as an antenna and radiate electromagnetic wave which is from the high frequency pulse current signal to GIS cavity. Electromagnetic wave is a form of electromagnetic fields motion and in the overlapping region of the electromagnetic field, the field strength at each point is the synthesis of field strength vector when the electromagnetic waves are excited alone.

UHF sensor is designed based on antenna theory and the mixed characteristics of received signals. Doublet antenna is used to take for a example. Assuming that electromagnetic wave 1 and 2 overlain region $\mathrm{D}$, the doublet antenna $l$ is in the region $\mathrm{D}, \mathrm{A}$ is a point on the antenna $l$, electric field intensities $\mathrm{E}_{1}, \mathrm{E}_{2}$ are generated by electromagnetic waves 1 and 2 at point A. The actual electric field strength of point $\mathrm{A}$ is $\boldsymbol{E}_{A}$. The axis of symmetrical doublet antenna is used to act as $z$-axis and the symmetry point of the axis is the origin point. Then the $x$-axis and $y$-axis can be defined by right-hand screw rule. The defined space Cartesian coordinate system is shown in Fig.1. $\vec{e}_{x} 、 \vec{e}_{y}$ and $\vec{e}_{z}$ is the direction vector.

$$
\left\{\begin{array}{l}
\boldsymbol{E}_{1}=e_{11}(t) \vec{e}_{x}+e_{12}(t) \vec{e}_{y}+e_{13}(t) \vec{e}_{z} \\
\boldsymbol{E}_{2}=e_{21}(t) \vec{e}_{x}+e_{22}(t) \vec{e}_{y}+e_{23}(t) \vec{e}_{z}
\end{array}\right.
$$

\footnotetext{
*Corresponding author: 15966635503@139.com
} 


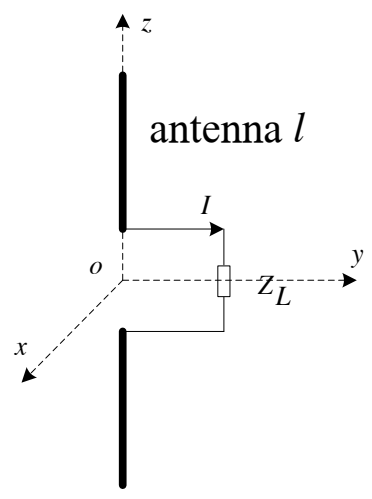

Fig.1. Schematic of doublet antenna and coordinate system.

According to the electromagnetic theory, only the electric field components which parallels to the axis of symmetrical doublet antenna can produce inductive electromotive force in the antenna and then the inductive currents are produced. The $z$-axis components of $\boldsymbol{E}_{1}, \boldsymbol{E}_{2}$ are $\boldsymbol{E}_{z 1}=e_{13}(t) 、 \boldsymbol{E}_{z 2}=e_{23}(t)$ respectively, and the inductive currents at the point $\mathrm{A}$ are $i_{1}(t)=e_{13}(t) / Z_{L}, \quad i_{2}(t)=e_{23}(t) / Z_{L}$. $Z_{L}$ is the impedance of the antenna.

$$
\begin{aligned}
\boldsymbol{E}_{\mathrm{A}} & =\boldsymbol{E}_{1}+\boldsymbol{E}_{2} \\
& =\left[e_{11}(t)+e_{21}(t)\right] \vec{e}_{x}+\left[e_{12}(t)+e_{22}(t)\right] \vec{e}_{y} \ldots \\
& +\left[e_{13}(t)+e_{23}(t)\right] \vec{e}_{z}
\end{aligned}
$$

When electromagnetic wave 1, 2 act together on the antenna, the current component which parallels to axis of symmetrical doublet antenna is

$$
i_{\mathrm{A}}(t)=\frac{E_{z \mathrm{~A}}}{\mathrm{Z}_{L}}=\frac{e_{13}(t)+e_{23}(t)}{\mathrm{Z}_{L}}=i_{1}(t)+i_{2}(t)
$$

It is shown that the mixed signals which are obtained by the antenna are the linear superposition of instantaneous value of electromagnetic wave 1,2 . When the nonlinearity of signal amplification and other auxiliary parts is ignored, the mixied signals acquired by UHF sensor are the sum of signals produced by two electromagnetic waves respectively.

\subsection{Mixing characteristics of PD signals}

When PD fault occurs in GIS, there are countless paths where excited electromagnetic waves reach UHF sensor. And the length and attenuation of each path is different. Representing the current signal of PD with $s(t)$, the current signal corresponding to the electromagnetic wave in each path is $a(\tau) s(t-\tau)$, where $\tau$ is the time delay, $0<\tau \leq$ $+\infty, a(\tau)$ is the attenuation coefficient. The mode of transmission is as shown in Fig. 2

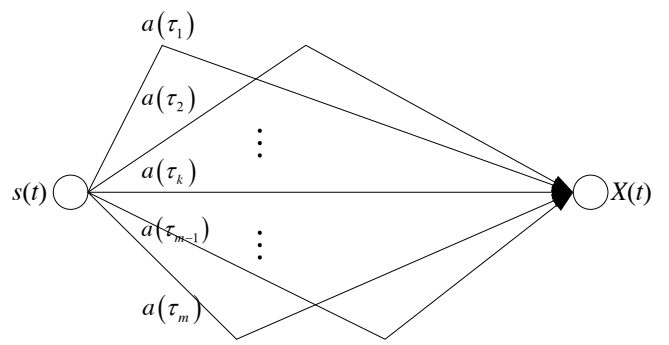

Fig.2. Propagation paths of Electromagnetic waves in GIS
According to the conclusions of section 2.1, the relationship between the single PD signal $x(k)$ obtained by UHF sensors and current signal $s(k)$ can be expressed as Eq.(4).

$$
x(k)=\sum_{\tau=0}^{\infty} a(\tau) s(k-\tau)=a(k) * s(k)
$$

Where, * represents the convolution operation, $a(k)$ represents impulse response from the source signal. The number of non-zero elements contained in $s(k)$ is far less than $x(k)$. It can be obtained from Eq. (4) that the single PD signal is the convolution of the discharge current signal and impulse response

If the number of independent discharge current signal sources in GIS is $n$, the mixed UHF PD signals $u_{j}(k)$ obtained by UHF sensor $j$ is given by

$$
\begin{aligned}
u_{j}(k) & =\sum_{i=1}^{n}\left[\sum_{\tau=0}^{\infty} a_{i j}(\tau) s_{i}(k-\tau)\right] \\
& =\sum_{i=1}^{n}\left[a_{i j}(k) * s_{i}(k)\right] \\
& =\sum_{i=1}^{n} x_{i j}(k)
\end{aligned}
$$

where $x_{i j}(\mathrm{k})$ is the single PD signal source $s_{i}$ obtained by sensor $j$. Vector $a_{i j}(k)$ is impulse response from PD signal source $s_{i}$ to sensor $j$. It is easy to conclude that the mixed PD signal is linear mixture between single PD signals. The mixed mode is as shown in Fig.3.

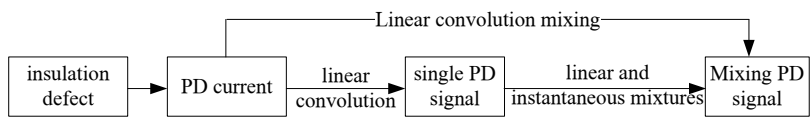

Fig.3. The mixing characteristics of UHF PD signals

Take white noise of the actual system into consideration, the Eq. (4) is rewritten as

$$
x(k)=a(k) * s(k)+g(k)
$$

where $g(k)$ is the additive noise. The Eq. (4) can be rewritten as

$$
u_{j}(k)=\sum_{i=1}^{n}\left[a_{i j}(k) * s_{i}(k)+g_{i j}(k)\right]=\sum_{i=1}^{n} x_{i j}(k)
$$

That is to say, the white noise signal will not change the mixturing characteristics of the PD signals.

\section{Inverse process of mixing process}

As shown in Fig.3, a single PD signal is the convolution of PD current signal and the corresponding impulse function. According to (4) and discrete signal deconvolution formula, the deconvolution can be got

$$
\left\{\begin{array}{l}
a(k)=\left[x(k)-\sum_{\tau=0}^{n u m} a(\tau) s(k-\tau)\right] / s(0) \\
s(k)=\left[x(k)-\sum_{\tau=0}^{n u m} s(\tau) a(k-\tau)\right] / a(0)
\end{array}\right.
$$

Where, $s(0)=x(0) / a(0), a(0)=x(0) / s(0)$, num is the discrete number of $x(k)$.

Mixed PD signals are linear mixture of single PD signals. According to (5), mixed PD signals are the sum of the single PD signal whose coefficient is "1". Because the distances from the same sensor to different PD points are different, the time for different sensors capturing 
single PD signals are different. When multiple PD faults coexisting, it can not be treated as the same signal. If the number of the PD current signals is $n$ and the number of UHF sensors is $m$, the number of single PD signal $x_{i j}$ is $m$ $\times n$. It is an over-complete blind source separation problem $[9,10]$.

UHF signal attenuates rapidly in sulfur hexafluoride gas, so the duration is only a few microseconds. The propagation distance is also limited. Therefore, the distance between sensors which capture the effective UHF signals is short. The positions between the sensors will be very compact for capturing signals produced by multiple insulation defects

The time differences among PD signals detected by UHF sensors can be ignored relative to the sampling intervals. So, $x_{i j}(k)$ in (5) can be expressed as

$$
x_{i j}(k) \approx c_{i j} x_{i}(k)
$$

Where, $x_{i j}(k)$ is the single PD signal of the PD current source $s_{i j}$, and $c_{i j}$ is a real coefficient. So the mixed signal can be expressed as

$$
u_{j}(k)=\sum_{i=1}^{n} x_{i j}(k) \approx \sum_{i=1}^{n}\left[c_{i j} x_{i}(k)\right]
$$

The number of single PD signals is $m \times n$. If $m \geq n$, it will be converted into the blind source separation problem based on the linear mixing model. Relevant researches have successfully achieved to isolate each single PD signal from mixed PD signals by using conventional blind source separation algorithm[11] . Every mixed way between PD signals and their inverse process are shown in Fig.4.

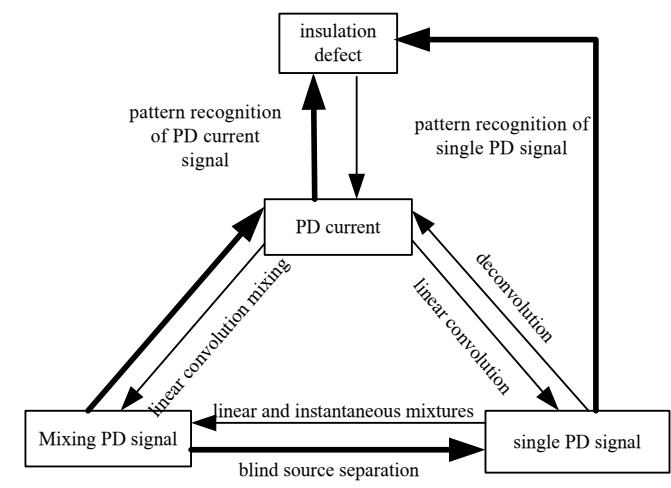

Fig.4. Mixing characteristics of PD signals and their reverse processes

As shown in Fig.4, there are two main ways for mixed PD signals pattern recognition: first, the mixed PD signals can be obtained by calculating blind deconvolution of mixed PD signals detected by UHF sensors; second, every PD signal can be gained by separeting the mixed PD signals with the blind source separation method. Because the blind source separation method is relatively mature, the second way is easier than the first way

\section{Simulation analysis}

In this paper, the XFDTD electromagnetic field software is used to test and verify the mixing characteristics of PD signals and the inverse process of mixing process mentioned above. A GIS straight barrel structure is built by the XFDTD whose structure is shown in Fig.5. The PD fault source is simulated as a monopole antenna, and the UHF sensor is assumed as a doublet antenna. The excitation sources $S_{1}$ and $S_{2}$ are both used as gauss pulse signals with $2 \mathrm{~ns} \mathrm{PWH}$, and $S_{2}$ is setted up with $100 \mathrm{~ns}$ delay.

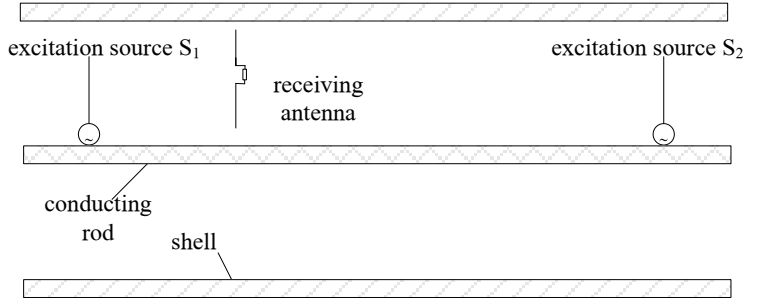

Fig.5. Cross section of GIS model
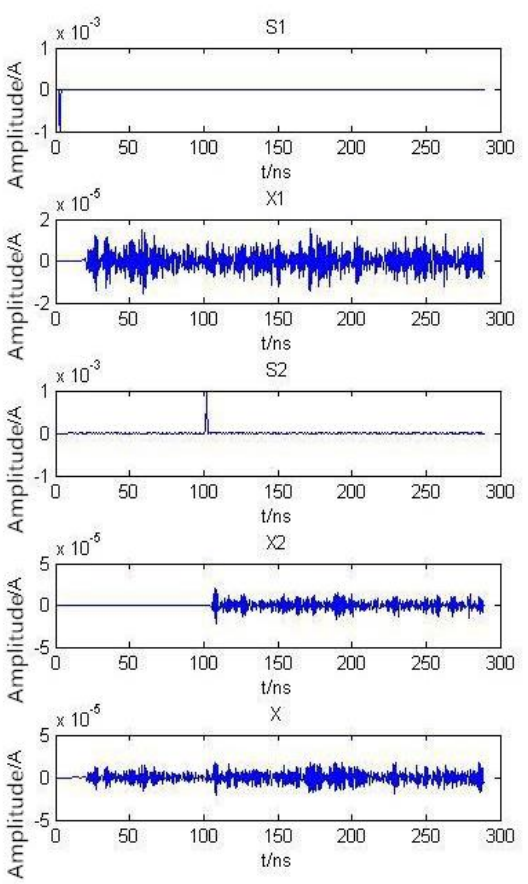

Fig.6. Simulation data of single and multiple PD sources

The excitation source waveforms and the obtained PD signals for a single source and the mixed sources are shown in Fig.6. In Fig.6, $S_{I}$ and $S_{2}$ are the waveforms of excitation sources 1,2 and $X_{1}, X_{2}$ are the PD signals detected by the receiving antenna for $S_{1}$ and $S_{2}$ respectively; $X$ is the mixed PD signal detected by the receiving antenna when $S_{1}$ and $S_{2}$ works in unison.

By comparing the excitation signal waveforms and corresponding detected signals, it can be concluded that the numeral gap of their nonzero sampling points is quite large as shown in (4). In other words, the sole PD signal is the convolution of the PD current signal and its corresponding impact response. Figure 7 shows the calculated results.

The corresponding comparison results of $X_{1}+X_{2}$ and $X$ are shown in Fig.8. It can be concluded that the waveforms of $X_{1}+X_{2}$ and $X$ are quite similar and the similarity factor is 0.9999999 . The signal to interference ratio between $X_{1}+X_{2}$ and $X$ is $261.2 \mathrm{~dB}$. Therefore, $X$ can 
be regarded as $X_{I}+X_{2}$. By the same way, a L structure is simulated and the simulation results are shown in Table 1.

All in all, the mixed partial discharge signals in GIS is the summation of every partial discharge signal. The single partial discharge signal is the convolution of the partial discharge current signal and its corresponding impact response. Therefore, the mixed partial discharge signals are the linear convolution of the partial discharge current signals.

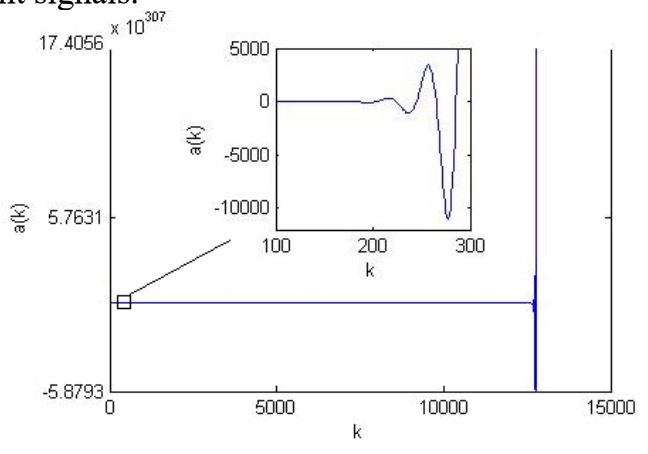

Fig.7. Result of deconvolution
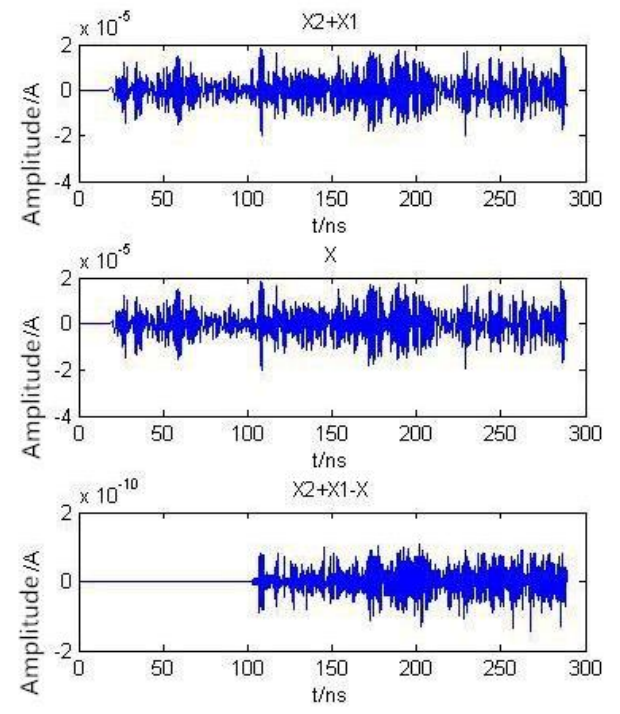

Fig.8. Comparison of $X_{1}+X_{2}$ and $X$

Table 1. Similarity of $X_{1}+X_{2}$ and $X$ in different structures

\begin{tabular}{|c|c|c|}
\hline Structure & $\begin{array}{c}\text { Similarity } \\
\text { factor }\end{array}$ & SIR(dB) \\
\hline $\begin{array}{c}\text { Straight barrel } \\
\text { (including } \\
\text { insulators) }\end{array}$ & 0.99999999977 & 261.2 \\
\hline L structure & 38 & 246.1 \\
\hline
\end{tabular}

\section{Conclusion}

The single partial discharge signal is the linear convolution of the partial discharge current signal and its corresponding impact response in GIS. The mixed partial discharge signals are the linear mixtures of every partial discharge signal.

There are two main ways for the pattern recognition of mixed PD signals: first, the mixed PD signals can be obtained by calculating blind deconvolution of mixed PD signals detected by UHF sensors; second, every PD signal can be gained by separeting the mixed PD signals with the blind source separation method. Because the blind source separation method is relatively mature, the second way is easier than the first way

\section{Acknowledgement}

We would like to express our gratitude to all those helped us during the writing and publsh of this paper..

\section{References}

1. J.Wang, Y. Qiu, Power System Technology, 23, pp.1-3, (2003).

2. M D Judd, O Farish, High Voltage Engineering Symposium, 467, pp.74-77(1999)

3. X. Huang, S. Kang, H. LI, et al. High Voltage Engineering, 32, pp.32-35(2006)

4. C. Yao, C. Huang, B. Wu, et al. High Voltage Engineering, 39, pp.272-279(2013)

5. J. Liu, M. Yao, C. Huang, et al. High Voltage Engineering, 35, pp.3458-2463(2009)

6. J. Liu, M. Yao, J. Wang, et al. Automation of Electric Power Systems, 32, pp.77-81, (2008)

7. Yang, M. Judd. IEE Proceedings Science Measurement and Technology,150, pp.119-127 (2003).

8. J. Tang, Q. Meng, C. Wang, et al. High Voltage Engineering, 36, pp.821-827(2010)

9. Z. Wang, P. Crossley, K. Cornick, et al. IEE Proceedings Science Measurement and Technology, 147, pp. 249-255(2000)

10. Yang, M. Judd. IEE Proceedings Science Measurement and Technology, 150, pp.119127(2003)

11. J. Tang, W. Wang, W. Li, et al. Journal of Chongqing University, 36, pp.1-8. (2013) 